\title{
Os transtornos da alimentação sob a ótica dos profissionais de enfermagem*
}

\author{
Eating disorders under the optics of nursing staff \\ Los trastornos de la conducta alimentaria bajo la óptica de profesionales de enfermería
}

\author{
Lucia Helena Grando ${ }^{1}$, Marli Alves Rolim ${ }^{2}$
}

\begin{abstract}
RESUMO
Objetivos: identificar as representações sociais da equipe de enfermagem acerca dos transtornos da alimentação. Métodos: pesquisa qualitativa, descritiva, exploratória, na qual foram entrevistados 12 profissionais, cujos relatos foram analisados sob a ótica da representação social. Resultados: os dados foram submetidos à análise de conteúdo, da qual emergiram duas categorias - "A construção do conhecimento" e "Campo das ações/sentimentos" que compreende temas como controle/limite, em que o sofrimento psíquico do trabalhador é mais evidente, principalmente na iminência do suicídio. Discussão: as representações sociais dos profissionais mostram-se ancoradas num discurso médico-científico, numa interface com o conhecimento prático da vivência cotidiana. Conclusão: precisamos atentar para o conteúdo afetivo-simbólico que os indivíduos imprimem nas relações e cuidar de todos os envolvidos nesse processo.

Descritores: Anorexia nervosa/enfermagem; Bulimia; Enfermagem psiquiátrica; Transtornos da alimentação
\end{abstract}

\begin{abstract}
Objectives: to identify the social representations of nursing staff regarding eating disorders. Methods: qualitative research, descriptive and exploratory, in which twelve professionals have been interviewed, and their reports have been analyzed under the social representation point of view. Results: the data were submitted a content analysis and two categories emerged - "Knowledge Construction" and "Actions/ Feelings Field", constituted by themes as control/limit, where the worker's psychic suffering is more evident, mostly those cases in imminence of suicide. Discussion: the mentioned knowledge gives us representations anchored essentially on a medical-scientific speech, in an interface with practical knowledge, which the daily life provides. Conclusion: it is necessary pay attention to affective-symbolic content of the relationships of nursing care process.
\end{abstract}

Keywords: Anorexia nervosa/nursing; Bulimia; Psychiatric nursing; Eating disorders

\section{RESUMEN}

Objetivos: identificar las representaciones sociales del equipo de enfermeros acerca de los trastornos de la conducta alimentaria. Métodos: pesquisa cualitativa, descriptiva, exploratoria, en la cual fueron entrevistados 12 profesionales, cuyos relatos fueron analizados bajo la óptica de la representación social. Resultados: la primera categoría de análisis, "La construcción del conocimiento" comprende síntomas físicos, psíquicos y factores psicosociales y la segunda, "Campo de las acciones/sentimientos", comprende temas como control /límite, donde el sufrimiento psíquico del trabajador es más evidente, sobretodo en la eminencia del suicidio. Discusiones: las representaciones sociales de los trabajadores muestran-se ancladas en un discurso médico científico, en una integración con el conocimiento práctico de la vivencia cotidiana. Conclusión: hay la necesidad de llamar la atención para el contenido afectivo-simbólico de las relaciones y cuidar de todos los enredados en estas.

Descriptores: Anorexia nerviosa/enfermería; Bulimia; Enfermería psiquiátrica; Trastornos de la conducta alimentaria

\footnotetext{
* Trabalho extraído da dissertação de mestrado: Representações sociais e transtornos alimentares: as faces do cuidar em enfermagem, apresentada na Escola de Enfermagem da Universidade de São Paulo - USP - São Paulo (SP), Brasil.

${ }^{1}$ Doutora em Enfermagem pela Escola de Enfermagem da Universidade de São Paulo USP - São Paulo (SP), Brasil; Assistente Técnica de Saúde III da Diretoria Executiva do Instituto de Psiquiatria do Hospital das Clínicas da Faculdade de Medicina da USP. Professora Adjunta da Universidade Guarulhos, Guarulhos (SP), Brasil.

${ }^{2}$ Professora Doutora do Departamento de Enfermagem Materno-Infantil e Psiquiátrica da Escola de Enfermagem da Universidade de São Paulo - USP - São Paulo (SP), Brasil.
} 


\section{INTRODUÇÃO}

A motivação para o presente estudo decorreu de nossa experiência profissional na qual nossa atenção se voltou para as pacientes que chegavam ao hospital em estado grave, magérrimas, correndo sério risco de vida, e que, apesar de caquéticas, insistiam que estavam gordas e, para perderem peso, mantinham regimes alimentares severos. Era intrigante ver aquelas garotas jovens, diagnosticadas como anoréxicas ou bulímicas, tão "destruídas" física e psiquicamente.

Estávamos diante de pessoas com características rígidas, de difícil relacionamento, manipulativas com relação ao tratamento. Tais características provocavam inúmeros sentimentos na equipe de enfermagem o que acabava por interferir na qualidade da assistência prestada, culminando, muitas vezes, em alta a pedido ou solicitação de mudança de local de trabalho por parte dos funcionários.

Eram comuns comentários críticos e a criação de verdadeiras “teorias" por parte dos profissionais que, não aceitando tais comportamentos, buscavam explicações baseadas em sua própria vivência pessoal.

Entendemos que a construção deste conhecimento, a partir da prática do cotidiano, caracteriza a produção de representações sociais, considerando que representação social é um conjunto de conceitos, explicações e afirmações que se originam na vida diária, no curso das comunicações interindividuais.

Por conseguinte, estudar as representações sociais que o pessoal de enfermagem tem acerca dos transtornos da alimentação e das pessoas portadoras dos mesmos, evidenciou-se como grande oportunidade de compreender a prática cotidiana.

Além disso, acreditamos que este estudo poderá trazer contribuições relevantes para a elaboração de futuros programas educativos, bem como fornecerá subsídios para discutir e refletir sobre a qualidade da assistência de enfermagem.

Delinearam-se, então, como objetivos da presente investigação: Identificar as representações sociais da equipe de enfermagem acerca da anorexia nervosa e bulimia e dos indivíduos portadores destes transtornos;

\section{Revisão de Literatura}

Dentre os transtornos da alimentação duas síndromes importantes são descritas: a anorexia nervosa e a bulimia $^{(1)}$.

A anorexia não é perda do apetite; trata-se de uma restrição alimentar deliberada, qualitativa e quantitativa: o adolescente segue um regime aberrante, ingere apenas quantidades ínfimas de alimentos escolhidos e reputados por seu baixo valor calórico. Essa procura incansável pela magreza é que leva a paciente a uma severa e autoinduzida perda de peso, para a qual finalidade usa diferentes e bizarros recursos ${ }^{(2-3)}$.

A perda de peso fica em torno de $15 \%$ ou mais do peso total e a redução extrema da ingestão alimentar pode chegar a um consumo de 500 a 700 calorias por dia, podendo ingerir menos que 200 calorias ou não comer por dias ${ }^{(4)}$. Afeta cerca de $1 \%$ a $2 \%$ da população feminina, entre as idades de 13 a 20 anos, mas pode ocorrer em qualquer grupo etário. Também é vista em homens, numa estimativa de apenas $5 \%$ a $10 \%$ da população com anorexia nervosa. A mortalidade foi estimada em $5,6 \%$ por década ${ }^{(5)}$.

Do ponto de vista psicopatológico, o que é característico da anorexia nervosa é a distorção da imagem corporal: apesar de muito emagrecida, a paciente percebe-se gorda, sente que algumas partes de seu corpo, como o abdômen, as coxas e as nádegas, estão muito gordas. O pavor de engordar persiste como uma idéia permanente, mesmo o indivíduo estando com seu peso bem abaixo do normal ${ }^{(6)}$.

Os critérios diagnósticos para a anorexia nervosa são: recusa a manter o peso corporal em um nível igual ou acima do mínimo normal adequado à idade e à altura; medo intenso de ganhar peso ou de se tornar gordo, mesmo estando com peso abaixo do normal; perturbação no modo de vivenciar o peso ou a forma do corpo, influência indevida do peso ou da forma do corpo sobre a auto-avaliação, ou negação do baixo peso atual; amenorréia, isto é, ausência de pelo menos três ciclos consecutivos após a menarca. Dois tipos são especificados: restritivo e compulsão periódica/ purgativo $^{(7)}$.

As causas da anorexia, embora imprecisas, mostram evidências da interação sócio-cultural, fatores biológicos, mecanismos psicológicos menos específicos e uma vulnerabilidade de personalidade, que contribuem para sua causação $\mathrm{O}^{(1)}$.

A bulimia é caracterizada pela ingestão de grande quantidade de alimentos muito rapidamente ("binge"), acompanhada de métodos compensatórios inadequados para controle do peso como vômitos, abuso de laxantes, diuréticos, dietas e exercícios físicos ${ }^{(8)}$. Sua incidência é de $2 \%$ a $4 \%$ da população feminina, com início entre 16 e 18 anos, numa proporção que vai de 1:6 a 1:10 (mulher: homem $)^{(4)}$. A estimativa de incidência ou prevalência desses transtornos varia dependendo da amostra e do método de avaliação(9).

Para a bulimia, segundo o DSM-IV, os critérios incluem: episódios recorrentes de compulsão periódica, caracterizados pela ingestão, em um período limitado de tempo (ex.2h), de quantidade de alimentos maior que a maioria das pessoas consumiria durante um período similar e sentimento de falta de controle sobre o 
comportamento alimentar durante o episódio; comportamento compensatório inadequado e recorrente, a fim de prevenir aumento de peso, como auto-indução de vômito, uso de laxantes, diuréticos, enemas ou outros medicamentos, jejuns ou exercícios excessivos; a compulsão periódica e os comportamentos compensatórios ocorrem, em média, pelo menos duas vezes por semana, por três meses; a auto-avaliação é indevidamente influenciada pela forma e peso do corpo. Inclui os tipos: purgativo e sem purgação ${ }^{(7)}$.

A morte pode ocorrer tanto na bulimia como na anorexia e são responsáveis pela maior taxa de letalidade, $20 \%$ entre todos os tipos de morbidades psíquicas ${ }^{(10)}$.

\section{Referencial teórico}

Elegemos a teoria das representações sociais como suporte teórico para a análise de conteúdo por considerar o mais apropriado para desvendar como os profissionais apreendem o indivíduo portador de transtorno alimentar e, em função dessa representação, como se organizam e orientam suas ações na prática cotidiana. Essa teoria parte do pressuposto de que o conhecimento é socialmente elaborado e compartilhado, tendo o objetivo prático de contribuir para a construção de uma realidade comum a um determinado grupo social, possibilitando a comunicação e a ação conjunta.

"Elas nos capacitam a classificar pessoas e objetos, a comparar e explicar comportamentos e os objetiva como parte do nosso cenário social’(11). Assim, as representações sociais permitem que o homem perceba o significado dos acontecimentos que ocorrem em sua vida cotidiana.

Nos dias de hoje, os meios de comunicação de massa abordam os padrões de beleza e determinam o modelo ideal de mulher; essa por sua vez assimila esses conceitos, muitas vezes tão integralmente que acaba por adoecer. A vida cotidiana se caracteriza por ser espontânea, com atividades automáticas e sem reflexão, como não teórica e as representações que os indivíduos fazem da realidade lhes dirigem e orientam o seu comportamento, ainda que não sejam percebidas como formações ideológicas ${ }^{(12)}$. Esse processo, a nosso ver, dirige e orienta a ação de pacientes com transtornos da alimentação, assim como as ações dos profissionais de enfermagem.

\section{MÉTODOS}

\section{Abordagem Metodológica}

Estudo qualitativo, de caráter descritivo e exploratório. A pesquisa qualitativa configurou-se como a mais pertinente, uma vez que ela não se preocupa em quantificar, mas em lograr explicar os meandros das relações sociais consideradas essência e resultado da atividade humana criadora, afetiva e racional, que pode ser apreendida através do cotidiano, da vivência, e da explicação do senso comum $^{(13)}$.

Teve como cenário as unidades que atendem indivíduos com transtorno alimentar do Instituto de Psiquiatria do Hospital das Clínicas da FMUSP. Este hospital tem por finalidade a assistência, o ensino e a pesquisa. Assim sendo, a veiculação do conhecimento científico se insere à prática, favorecendo a formação das representações sociais através da imbricação do conhecimento científico com o senso comum, universo "reificado" e consensual.

O número de sujeitos não foi definido a priori pois, segundo a abordagem qualitativa ${ }^{(12)}$, a análise das descrições foi sendo processada até que ocorresse invariância dos dados. Portanto, fizeram parte do estudo seis enfermeiros, um técnico de enfermagem e cinco auxiliares de enfermagem envolvidos no cuidado de pacientes com transtornos da alimentação.

A coleta de dados foi realizada no período de novembro de 1998 a março de 1999, por meio de entrevista realizada em local privativo, na própria instituição, orientada por roteiro (Anexo I).

O projeto foi submetido à "Comissão de Ética para Análise de Projetos de Pesquisa" do Hospital das Clínicas, de acordo com a Resolução no 196/96 do Conselho Nacional de Saúde, sendo aprovado. Os sujeitos assinaram o Termo de Consentimento Livre e Esclarecido.

Os dados foram submetidos à técnica de análise de conteúdo segundo Bardin ${ }^{(14)}$, mais especificamente a temática, tendo a teoria moscoviciana como suporte teórico de análise. Após a organização do material discursivo, optamos por seguir as etapas: pré-análise, exploração do material, tratamento dos resultados obtidos e interpretação ${ }^{(15)}$.

\section{RESULTADOS}

Os sujeitos e suas representações

A partir dos relatos dos profissionais, foi possível identificar aspectos importantes dessa população, as representações sociais que construíram acerca dos transtornos da alimentação, na busca de elementos particulares que pudessem deixar transparecer como essas representações são construídas e como influenciam as ações que desenvolvem no processo de cuidar, objetivos últimos da presente pesquisa.

Dessa maneira, surge uma forma singular de cuidar, ancorada em conhecimentos prévios, já estabelecidos e oriundos de fontes teóricas, da prática diária, de conceitos e preconceitos sociais, estigmatizantes em maior ou menor grau, em crenças e vivências pessoais.

O primeiro núcleo de convergência de significados que se delineou foi com relação à concepção dos transtornos da alimentação que deu origem à categoria 
"A Construção do Conhecimento". Os sujeitos explicitam essa concepção descrevendo comportamentos nos quais identificam sintomas físicos, psíquicos e demandas psicossociais. O conhecimento socialmente elaborado e compartilhado mostra-se ancorado no discurso médico veiculado na instituição (conhecimento científico), esquemas já constituídos com base essencialmente organicista, numa interface com o conhecimento prático (senso comum) que a vivência cotidiana possibilita.

É a partir dos sintomas físicos que os sujeitos, inicialmente, localizam os transtornos da alimentação no corpo do indivíduo.

"É emagrecida, com fisionomia de mais idade, envelhecida. Ficam parecendo, quando sem roupa, umas caveirinhas, mesmo!!! Dá pra contar as costelas".

"A bulimia é uma patologia onde a pessoa tem uma compulsão alimentar e provoca vômito..., tem dificuldade em alimentar-se, apresenta queixas constantes de obstipação intestinal, tem amenorréia, são resistentes ao tratamento, não se consideram doentes. Têm rituais... Têm uma infinidade de coisas...".

Esta ancoragem da compreensão dos transtornos da alimentação no modelo organicista, o qual determina a causa das patologias no corpo e institui suas terapêuticas somáticas, também foi evidenciada em estudo da representação social que os profissionais de enfermagem têm da doença mental, mostrando-a com um núcleo figurativo que aponta o cérebro como instância biológica de localização da doença ${ }^{(16)}$.

É também a partir do aspecto físico, da magreza intensa, da gravidade e do risco de morte, que os entrevistados fazem a diferenciação entre os dois quadros: anorexia e bulimia. Os sujeitos consideram a anorexia mais grave que a bulimia. Na realidade, ambas apresentam o mesmo grau de gravidade e risco de morte.

Por outro lado, as representações que os sujeitos têm acerca das alterações de comportamento refletem um descontrole que se manifesta na esfera alimentar, com condutas extremas que oscilam do jejum ao comer em exagero (binge). Essa forma de explicar estes transtornos como sendo um descontrole é uma representação social já identificada por outros pesquisadores, com relação à doença mental de uma forma geral( ${ }^{(17-18)}$.

Com relação aos sintomas psíquicos, a distorção da imagem corporal aparece de forma enfática, assim como características de comportamento manipulativo, autodestrutivo, carência, baixo limiar à frustração e necessidade de chamar a atenção.

"A anorexia é um transtorno alimentar em que a pessoa, principalmente mulher, tem uma imagem distorcida de sua própria pessoa física e não quer saber de se alimentar".

"Bulimia também é um transtorno alimentar, psíquico e que, prá mim, é uma auto-agressão. O que mais chama a atenção é a auto-agressão, meio velada. Elas estão sempre bem, mas estão ali, se detonando, se auto-agredindo mesmo".

Os depoentes concebem também esses transtornos ancorados numa perspectiva social, na qual está embutido um padrão estético determinado culturalmente e veiculado pela mídia, e a influência da família enquanto um grupo social primário. Consideram que a sociedade, através da imposição de padrões estéticos de beleza, vai influenciar as profissões nas quais as pessoas usam o próprio corpo como seu instrumento de trabalho.

"A causa é social, porque além das pessoas se alimentarem de maneira inadequada, ela se priva do que é saudável para estar sempre magra. Aqui também entram as questões dos exercícios, academias. A imagem que épassada, de estereótipo, de tipo fisico, é ser magra. Um exemplo disso é que todas as modelos são magras, com aspecto de doente, como as da FORUM".

Retomando a questão da influência da família “a conduta humana só pode ser explicada em função dos vínculos que o sujeito estabelece com o ambiente"(19).

Verificamos que os valores e crenças da família influenciaram, de forma direta ou indireta, a origem do transtorno, interferindo na construção da imagem corporal.

E é nessa família que os sujeitos identificam inicialmente a falta de limites, fator este que os entrevistados apontam como algo que permeará os relacionamentos entre os pacientes e os profissionais, causando uma série de sentimentos desagradáveis.

Estudiosos apontam que a psicodinâmica da família interfere na doença, o que torna muito difícil cuidar apenas da paciente sem inserir o contexto familiar; dessa forma, as intervenções familiares, o aconselhamento e o apoio se tornam necessários, tanto para a paciente quanto para os familiares ${ }^{(20)}$.

A segunda categoria de análise "Campo de Ações/ sentimentos" compreende temas como limite/controle que representam o processo de cuidar destes pacientes e onde se evidencia a grande carga de sofrimento psíquico do trabalhador.

"É complicado, né! Criança é mais complicado ainda. Aqui, com as adultas, tem que ter uma conduta mais rígida, muita vigilância para não provocar vômito após as refeições".

Identificamos aqui o conflito que o profissional enfrenta quando percebe a dificuldade que os pacientes experimentam em tomar decisões, em ter controle sobre sua própria vida, colocando-a em risco muitas vezes, o que leva o profissional a uma postura de limite/controle, considerada excessiva por ele próprio, pois não redunda 
em amadurecimento por parte do paciente, que continua sem possibilidades de vivenciar uma verdadeira autonomia.

É principalmente o risco de suicídio que provoca sentimentos muito fortes e contraditórios, gerando desgaste emocional intenso no trabalhador de enfermagem.

"Teve uma vez que en a abordei para parar de fazer os exercícios e ela disse que a doença era mais forte que ela. Aquele dia en fiquei mal, achei que devia pegar mais leve. Ela acabou morrendo lá fora. A gente acha que o tratamento não está adequado..., se vale mesmo a pena ficar no pé..., acho que quando a paciente está ruim não é legal isso aí...”.

Os profissionais, para evitar a tentativa de suicídio, assumem uma postura controladora, e para se protegerem do estresse, utilizam mecanismos de defesa, como a fragmentação do relacionamento enfermeira-paciente, despersonalização, categorização e negação da importância do indivíduo, distanciamento e negação de sentimento ${ }^{(21)}$.

\section{DISCUSSÃO}

As concepções destes transtornos fortemente impregnadas por um senso de descontrole e por características como comportamento manipulativo, autodestrutivo, de baixo limiar à frustração e de necessidade de chamar a atenção, mostram o cuidar que aparece ainda fortemente representado pelo limite/ controle. Porém, começa a despontar uma intencionalidade do cuidar permeada por atitude de acolhimento, quando as ações envolvem escuta, estabelecimento de vínculo de confiança e necessidade de respeito à individualidade de cada paciente.

O cuidado apresenta-se, pois, preocupado com as questões da subjetividade do indivíduo e, portanto, permeado pela afetividade, quando os profissionais demonstram sua preocupação que ultrapassa a percepção meramente física, na busca da compreensão do sofrimento psíquico dos sujeitos.

"Orientar e favorecer as relações afetivas e os vínculos sociais do doente mental deveria ser um compromisso real dos profissionais de saúde mental’'(18). É interessante notar que quando estabelecem o vínculo de confiança com as pacientes, é que expressam sua satisfação e prazer no trabalho.

Reconhecemos que "para se promover a saúde não basta ensinar novos conhecimentos e padrões, é preciso compreender os motivos e emoções que medeiam tais conhecimentos e práticas, desvelando a base afetivovolitiva do agir e pensar. Em outras palavras, as relações, a consciência e as ações não são apenas cognitivas e sociais; elas têm carga afetivo-simbólica" (22).

O enfermeiro, como agente de saúde e educador, atua como intermediário entre o conhecimento científico e o senso comum, portanto, deve ampliar suas dimensões do cuidar, buscando estratégias que privilegiem o paciente, seu sofrimento, sua dor. O desafio deste profissional é trabalhar com essas pessoas para que reavaliem suas próprias condutas e as tornem mais flexíveis, de modo a buscar adaptação criteriosa que lhes permita vivenciar um grau de autonomia saudável ${ }^{(23)}$. Consideramos que "jamais podemos nos esquecer do aspecto subjetivo do ser humano, de tratar cada um como único, de ser os que abrigam, que compreendem e apreendem o que o outro tem para ensinar. Pois, como cuidar de quem não conhecemos? ${ }^{(24)}$

\section{CONCLUSÃO}

Concluímos que os achados obtidos neste estudo estão em estreita relação com a necessidade de novas dimensões do cuidar e nos propiciaram uma compreensão mais profunda de nossas ações, enquanto enfermeiras e educadoras.

Buscar compreender as representações dos profissionais, conhecer e compreender a trama onde eles e as pacientes estão inseridos, as ações cotidianas e os sentimentos subjacentes, é uma tarefa bastante difícil. Novos caminhos poderão ser trilhados na busca de uma assistência que privilegie o ser humano em sofrimento psíquico. É preciso dirigir nossa atenção para o conteúdo afetivo-simbólico que os indivíduos imprimem em suas relações, e cuidar de todos os envolvidos nessa rede de relacionamentos.

\section{REFERÊNCIAS}

1. Organização Mundial de Saúde - OMS. Classificação Estatística Internacional de Doenças e Problemas Relacionados à Saúde - CID-10. São Paulo: EDUSP; 1996.

2. Hevzey MFL. Psychopathologie de l'adolescent. Rev Infirm. 1988; 38(20): 19-21.

3. Cordás TA, Vallim Júnior A. Fome de cão: Quando o medo de ficar gordo vira doença: anorexia, bulimia, obesidade. São Paulo: Maltese; 1993. p.17-27.

4. Stuart GW, Sundeen SJ. Pocket guide to psychiatric nursing. St. Louis: Mosby; 1995. p. 357-74.

5. Cochrane CE. Respostas de regulagem alimentar e transtornos alimentares. In: Stuart GW, Laraia MT. Enfermagem psiquiátrica: princípios e prática. 6a ed. Porto Alegre: Artmed; 2001. p.559-80.

6. Dalgalarrondo P. Psicopatologia e semiologia dos transtornos mentais. Porto Alegre: Artes Médicas; 2000.

7. Manual diagnóstico e estatístico de transtornos mentais: DSM-IV. 4a ed. Traduzido por Batista D. Porto Alegre: Artes Médicas; 1995. p. 511-22.

8. Salzano FT, Cordás TA Transtornos da Alimentação. In: 
Abreu CN, Salzano FT, Vasques F, Cangelli Filho R, Cordás TA, organizadores. Síndromes psiquiátricas: diagnóstico e entrevista para profissionais de saúde. Porto Alegre: Artmed; 2006. p.111-7.

9. American Psychiatric Association. Practice guidelines for the treatment of patients with eating disorders. 2nd ed. Washington, DC: American Psychiatric Press; 2002.

10. Nunes MAA, Ramos DC. Anorexia nervosa: classificação diagnóstica e quadro clínico. Transtornos alimentares e obesidade. In: Nunes MAA, Appolinário JC, Abuchaim ALG, Coutinho W, Ramos DC, Azevedo AMC, et al. Transtornos alimentares e obesidade. Porto Alegre: Artmed; 1998. p.21-30.

11. Moscovici S. Notes towards a description of social representations. Eur J Soc Psychol. 1988; 18:211-50.

12. Salles LMF. Representação social e cotidiano. Didática. 1991; (26/27): 11-20.

13. Minayo MCS. O desafio do conhecimento: metodologia da pesquisa social qualitativa em saúde [tese]. Rio de Janeiro: Escola Nacional de Saúde Pública; 1989.

14. Bardin L. Análise de conteúdo. Lisboa: Edições 70; 1994.

15. Gomes R. A análise de dados em pesquisa qualitativa. In: Minayo MCS, Deslandes SF, Otávio Cruz Neto. Pesquisa social - teoria, método e criatividade. Petrópolis: Vozes; 1998. p.67-80.

16. Rolim MA. Representações sociais acerca do doente mental atendido em emergências psiquiátricas [tese]. São Paulo:
Escola de Enfermagem da Universidade de São Paulo; 1993.

17. Scazufca M. Usuário de calmantes: um estudo das representações mentais de pacientes atendidos em ambulatórios de saúde mental da Secretaria de Estado de Saúde de São Paulo [tese]. São Paulo: Instituto de Psicologia da Universidade de São Paulo; 1990.

18. Machado AL. Espaços de representação da loucura: religião e psiquiatria [tese]. São Paulo: Escola de Enfermagem da Universidade de São Paulo; 1999.

19. Shimizu HE. Sofrimento e prazer no trabalho vivenciado pelas enfermeiras que trabalham em unidade de terapia intensiva em um hospital-escola [tese]. São Paulo: Escola de Enfermagem da Universidade de São Paulo; 1996.

20. Lupo A. Terapia familiar. In: Busse SR, organizador. Anorexia, bulimia e obesidade. Barueri: Manole; 2004. p.281-97.

21. Menzies I. O funcionamento das organizações como sistemas sociais de defesa contra a ansiedade [mimeografado]. São Paulo: Fundação Getúlio Vargas, s.d.

22. Sawaia BB. Análise psicossocial do processo saúde-doença. Rev Esc Enfermagem USP. 1994; 28(1):105-10.

23. Grando LH, Rolim MA. Família e transtornos alimentares: as representações dos profissionais de enfermagem de uma instituição universitária de atenção à saúde mental. Rev Latinoam Enfermagem. 2005; 13(6): 989-95.

24. Corbani NMS. O dilema conceitual ético do enfermeiro: como cuidar de quem não conhecemos? Acta Paul Enfermagem. 2004; 17(4):445-9.

\begin{abstract}
ANEXO I
Perfil do entrevistado: iniciais, idade, sexo, escolaridade/formação, tempo de formado, função atual, tempo de trabalho nessa Instituição, experiência anterior em psiquiatria e tempo de trabalho, nessa enfermaria, com pacientes com transtornos alimentares.

Questões: Teve algum tipo de treinamento para trabalhar com pacientes anoréxicos e bulímicos? Sentiu falta de preparo para trabalhar com eles? Para você o que é anorexia nervosa? Para você o que é bulimia? Que tipo de pessoa você acha que ficaria com maior facilidade com esses transtornos? Descreva como é uma pessoa com transtorno alimentar? Quais as causas que você atribui ao transtorno alimentar? Como o paciente com esse transtorno deve ser tratado? Acha que a pessoa com esse transtorno tem cura? O que sente quando está perto de um paciente com esse transtorno? Para você, quais as características necessárias para trabalhar com esse tipo de paciente? Qual é a sua atuação junto aos pacientes com transtorno alimentar? O que faz? Como você percebe a influência da família em todo o processo de desenvolvimento dos transtornos alimentares? Fale de um caso mais agradável e um mais difícil.
\end{abstract}

